\title{
Multi-scale Structure Formation and Dynamics in Cosmic Plasmas
}

\author{
A. Balogh ${ }^{1}$ A. Bykov ${ }^{2,3,4} \cdot$ J. Eastwood ${ }^{5} \cdot$ J. Kaastra $^{6}$
}

Received: 12 February 2015 / Accepted: 13 February 2015 / Published online: 3 March 2015

(C) Springer Science+Business Media Dordrecht 2015

\section{Plasma Structures: From the Earth Magnetosphere to Clusters of Galaxies}

Being statistically homogeneous on cosmological scales the Universe is demonstrating a very rich variety of structures and components at smaller scales. Gravity is the source of energy and the driving force for formation of the large scale structure (LSS), clusters of galaxies, galaxies and stars, while the electromagnetic fields are drastically important for the microphysics of the momentum and energy transfer in the baryonic matter and radiation. The Workshop "Multi-scale structure formation and dynamics in cosmic plasmas" which was held at International Space Science Institute in April 2013 was devoted to a broad discussion of different aspects of formation, dynamics, and observational appearance

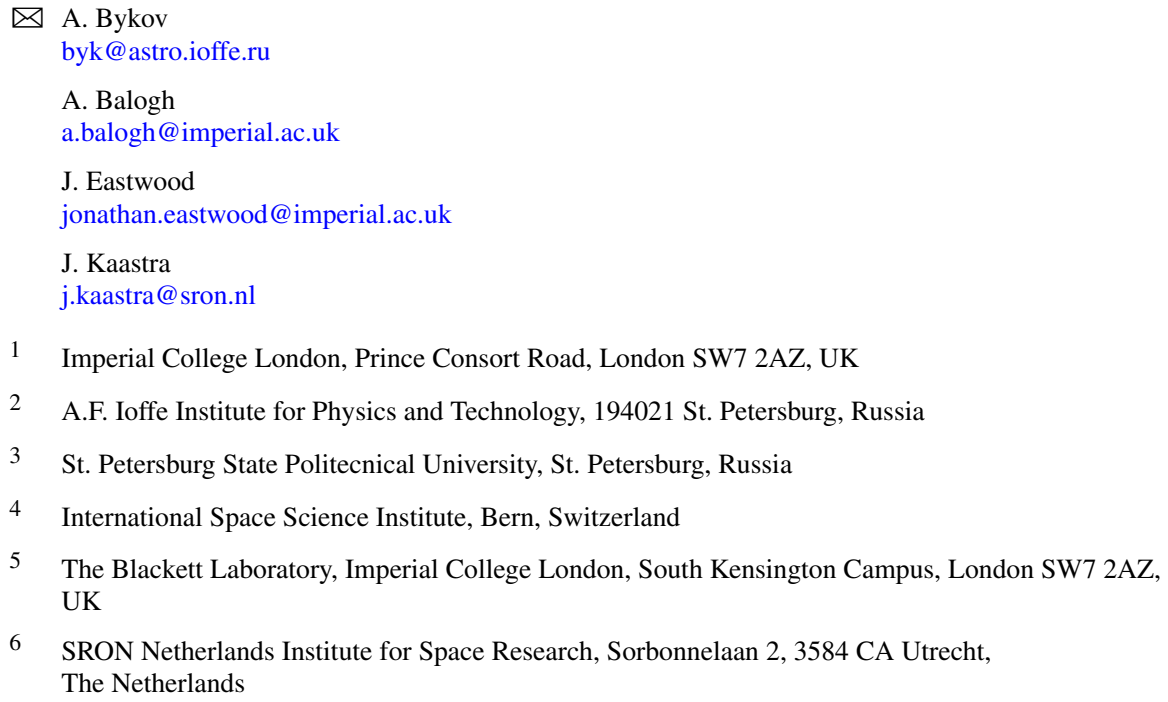

6 SRON Netherlands Institute for Space Research, Sorbonnelaan 2, 3584 CA Utrecht, The Netherlands 
of plasma structures at different scales ranging from LSS to the Earth's magnetosphere. The present book is composed from the reviews, which are based on the discussions at the ISSI Workshop. It contains review papers on the basic processes of structure formation in cosmic plasmas starting from electric currents, which produce magnetic structures in planet magnetospheres, stellar winds, and relativistic plasma outflows like pulsar wind nebulae and Active Galactic Nuclei jets. The important role of the helicity concept on the structure formation and evolution of the large scale magnetic fields in highly conductive cosmic plasmas is emphasized in the book. Microscopic dynamics of plasma flows and magnetic fields was discussed in depth in Space Sciences Series of ISSI, Volume 47 "Microphysics of Cosmic Plasmas", originally published as Space Science Reviews (Balogh et al. 2013), and thus, it is highly recommended to refer to that volume while reading the present volume. Cosmological aspects of plasma structures are reviewed within a discussion of large-scale structure formation from the first non-linear objects to massive galaxy clusters, which is followed by a review of observations and current models of structures and components in galaxy clusters. Supernova remnants interacting with molecular clouds are among the most important ingredients of the global galactic ecology with a profound effect on the phenomena related to star formation. The multi-wavelength view from the radio to gamma-rays with modern high resolution telescopes revealed a beautiful and highly informative picture of both coherent and chaotic plasma structures tightly connected by strong mutual influence. The same plasma processes are likely to control the structure and dynamics of Earth's magnetosphere where detailed direct satellite observations are available. The properties of magnetic field fluctuations and structures in the outer solar atmosphere and Earth's magneto-tail, which have direct implications for the general problem of structure formation in hot plasmas, are discussed in depth in the volume.

This volume is aimed at graduate students and researchers active in the areas of astrophysics and space science.

Acknowledgements The Editors are greatly indebted to all the participants of the Workshop held in ISSI Bern on 15 to 19 April 2013 who brought their broad range of expertise and interest in the astrophysics of plasmas to discuss the vast range of scales of plasma structures in the Universe and how the study of their formation and dynamics can illuminate processes at the different scales. We thank the staff of ISSI for their dedicated support: Prof. Rafael Rodrigo, Executive Director, and his colleagues Prof. Rudolf von Steiger, Jennifer Fankhauser, Andrea Fischer, Saliba Saliba and Sylvia Wenger. The resulting collection of review papers was the outcome of the exchanges and fruitful collaboration among the participants; we thank them for their successful efforts to integrate the lessons learned in the different topics, as the reviews in the volume testify. Thanks are also due to the reviewers of the papers; in all cases the reviews were thorough and constructive and the volume bears witness to their contribution. Finally the Editors thank the staff of Space Science Reviews, as well as the production staff for their patience on occasion and for an excellently produced volume.

\section{References}

A. Balogh, A. Bykov, P. Cargill, R. Dendy, T. Dudok de Wit, J. Raymond, Microphysics of cosmic plasmas: background, motivation and objectives. Space Sci. Rev. 178, 77-80 (2013). doi:10.1007/s11214013-0027-1 